\title{
Part IV: Decisions to forgo life-sustaining treatment under conditions of scarcity
}

The preceding guidelines have addressed decisions to forgo life-prolonging medical treatments as if scarcity were not a problem. However, growing needs and demands, a growing range of increasingly costly medical options, and limited resources compel us to recognise that decisions, made necessary by scarcity, to forgo treatments that are both desired and beneficial is increasingly a troublesome fact of clinical life and experience $(1,2)$. Many of these decisions will be about patients who are near the end of their lives where options tend to be most costly, marginal benefit difficult to evaluate and decisions final. The decisions may be either necessitated by an absolute scarcity, as when sufficient organs for transplant are simply unavailable, or, more subtly, by a need to choose between competing claims, as when a procedure is considered too costly in relation to its expected benefits and opportunity costs by those who provide the resources and those who manage them.

The first three sections of these guidelines provide guidance regarding conflicts that arise between patient autonomy and professional beneficence, occasionally over-zealous or misguided and sometimes in conflict with other forces. When scarcity is recognised as an element in decision-making, new conflicts are revealed: those between justice and efficiency on the one hand and patient autonomy, now often in concert with professional beneficence, on the other. These conflicts are especially difficult for clinicians because not only are they more complex, touching in significant ways several different levels of decision-making that necessarily mutually affect one another, but they are also uncomfortable, nearly always involving combinations of the most difficult kinds of ethical decisions: those that involve degrees of uncertainty hard to measure or understand and those that require weighing the well-being of an aggregate of individuals (society, community or members of the same health plan) against the well-being of an individual patient.

To assist in the difficult task of making a responsible treatment or allocation decision at one level that is not counter-productive at another level, the following interrelated levels of decision-making have been distinguished. The guidelines that follow are organised according to these levels.

\section{Levels of decision-making}

a) The aggregate level: Decisions at the national or regional level about the allocation of resources to health care as a whole or for particular categories of patients. Decisions at this level shape and define the conditions of scarcity under which subsequent decisions to treat or not to treat must be made.

b) The intermediate level: Decisions about allocation of existing health-care resources within health-care systems, health plans and institutions such as hospitals, clinics, health centres.

c) The individual level: Decisions within health-care institutions, including those at the bedside, about whether or not a specific treatment should be initiated or continued.

Consideration of justice or efficiency will be dominant at the aggregate and, to a somewhat lesser extent, the intermediate levels; consideration of autonomy and respect for persons and professional integrity and beneficence will be dominant at the individual and intermediate levels. Each of these considerations will have some influence at each level.

Our deliberations on the particular set of issues that emerge when non-treatment decisions are considered in light of scarcity have centred around two foci:

- Understanding the ethical demands of justice and efficiency under conditions of scarcity and

- Understanding the conflicts between the demands of justice and efficiency and the demands of autonomy and beneficence.

\section{Understanding the ethical demands of justice and efficiency under conditions of scarcity}

\section{JUSTICE}

A widely accepted understanding of the principle of justice requires equality of treatment for those who are in all relevant respects equal, and permits inequality of treatment only when justified by, and commensurate with, ethically relevant inequalities. Although the 'respects' deemed ethically relevant will vary in different contexts, cultures, groups and individuals, there are four ethical dimensions that should be considered by any society, institution, or individual in 
determining which 'respects' are relevant to justify unequal health care:

1) people's health-care needs, including the capacity of people to benefit from treatment,

2) people's rights to control their own possessions, including their accumulated wealth,

3) the effect of the decision to administer or withhold treatment on the total benefit or welfare of the community,

4) the role, if any, that merit and demerit should play in these decisions.

Any just system of allocating scarce medical resources should include respect for these ethical dimensions and consideration of the sometimes delicate balance that must be maintained among them.

\section{Need}

Health-care need has been interpreted to mean different things by different people $(3,4)$. Regardless of how health-care need is defined, it is widely agreed that the greater the need, the greater should be the moral imperative that the need be met. Inevitably, however, some needs cannot be met and it is also widely agreed that health care should, in conditions of scarcity, be made available to an individual only if it would contribute positively towards meeting the need of that individual with an acceptable probability of success. When it is not possible to meet the needs of all individuals out of the available resources, respect for the ethical dimension of need would suggest that available resources be distributed in proportion to need. Such distribution will conflict with respect for the ethical dimension of property (see number 2 below) and, depending on which definition of need is used, may well conflict with a desire to distribute health-care resources in a way that maximises the total healthbenefit of society.

\section{Property}

Respect for people's control over their justly acquired possessions or 'holdings', including their income and accumulated wealth, derives from respect for autonomy. Respect for this ethical dimension inevitably conflicts with respect for need. Those in need will want maximal resources to meet their need but those who pay, through systems of private or social insurance, for the provision of medical-care resources for others will want, at the very least, to set reasonable limits to their contributions. Moreover, in a given health-care system, the ability of those in lesser need to purchase scarce life-prolonging resources will inevitably deny life-prolonging resources to others in greater need, but with less ability to pay. This conflict between respect for need and respect for property is a classical conflict in any social-welfare system (5). It is, however, particularly important in health care because individual need and wealth are often inversely related.

\section{Total benefit of society}

Respect for the total benefit of society would require? that whenever medical procedures that could be $\vec{F}$ beneficial to individuals have to be limited because of $\frac{?}{\circ}$ scarcity, available resources should be allocated among $\frac{\mathrm{C}}{\mathrm{O}}$ persons so that they produce the greatest overall $\bar{c}$. utility. Such allocation inevitably conflicts with respect $\overparen{\Phi}$ for the ethical dimensions of both need and property $\bigcirc$ (6).

\section{Merit}

Normally, social status and social merit are not $\vec{\omega}$ appropriate criteria for the allocation of scarce health- $\frac{\Omega}{3}$ care resources. It has, however, been suggested that $\stackrel{\nabla}{\circ}$ certain forms of 'demerit' might be responsibly applied $\vec{\infty}$ to some allocation decisions. For example, people $i s$ whose needs for care arise in whole or in part from their $\frac{-}{0}$ own 'fault' (for example, from excessive smoking, drug? abuse, participation in dangerous sports, or non- $\vec{\sigma}$ compliance with prescribed treatment) could be음 required to make a larger than normal contribution to the cost of whatever care they receive (7).

\section{EFFICIENCY}

The maxim of efficiency follows from the principles of $\frac{3}{\Phi}$ non-maleficence and beneficence. It requires that $a \stackrel{\Phi}{~}$ given expected outcome of any medical procedure, $\vec{\oplus}$ including a life-prolonging procedure, whether in respect to an identified patient or to groups anonymous patients, ought to be achieved at the least opportunity-cost (8). The force of the principle derives from the fact that, should the principle not be met, fewer resources would be available, either immediately $\bar{\Phi}$ or at some future date, for accomplishing other desired and ethical outcomes.

At the basic level of the doctor-patient relationship, when resources available to the doctor are limited, efficiency requires that resources ought not to be employed if the benefits to a particular patient are $\overline{\bar{S}}$ judged to be less than the benefits denied to others for $\frac{\Phi}{\sigma}$ whom the same doctor has responsibility. At higher 3 . levels, such as hospital management, the principle is equally applicable in determining the terms, entitlement, facilities, supplies or resource budgets ${ }_{\bigcirc}$ which, in turn, constrain doctors' choices at the more basic level.

\section{Understanding the conflicts between the demands of justice and efficiency and the demands of autonomy and beneficence}

Understanding the demands of justice and efficiency $\sigma$ and sorting out the conflicts that arise between them is not, however, sufficient preparation for responsible ethical decision-making under conditions of scarcity. The demands of justice and efficiency will inevitably conflict both with concern for patient autonomy and with the professional application of appropriate $\vec{D}$ beneficence. It is important to think clearly and $\frac{\rho}{\mathbb{Q}}$ candidly about these conflicts in advance of difficult $\stackrel{\varrho}{2}$ clinical situations. A great deal can be accomplished by 
education of both health-care professionals and the general public about the nature of these conflicts and the inevitable need for compromise in sorting them out. Finally, however, education and understanding of the conflicts is not enough. Individuals will vary in their inclinations and abilities to adjust voluntarily their autonomous desires to the collective interest. At times more than voluntary adjustment will be required. The health-care system of any society must have in place a fair and reliable mechanism to explain openly and to enforce justly such adjustments.

The guidelines that follow are not offered as a solution to all of these conflicts. They do, however, focus on major ethical considerations, applicable at several levels of decision-making in a wide variety of situations, which can be used to guide any allocation decision that is intended to be both just and efficient and any non-treatment decision, made under conditions of scarcity, that is intended to weigh responsibly the demands of justice and efficiency against those of respect for autonomy, nonmaleficence, and beneficence. They may also be used to appraise the degree of justice and efficiency in existing allocations.

\section{Guidelines (9)}

GENERAL GUIDELINES APPLICABLE TO DECISIONS AT ALL THREE LEVELS

1. There ought to be general and open discussion of the ethical principles used to guide decisions at all levels regarding either the allocation of health-care resources or the limiting of treatment under conditions of scarcity (10). Widespread dissemination of such principles or guidelines to decision-makers at all levels and to the general public should be routine.

2. Prompt dissemination of accurate information about efficacy, effectiveness and cost-effectiveness ought to be routinely available to all those who have responsibility either for resource-allocation decisions or for clinical-treatment decisions under conditions of scarcity (11).

3. Decision-makers at all levels ought to keep themselves informed regarding the evidence for efficacy, effectiveness and cost-effectiveness and, having due regard for the context in question, act upon this information. Guidelines and protocols for applying the results of these studies to specific cases should be established by appropriate institutions and professional groups.

4. The need for effective treatment must be a dominant consideration in determining who receives care and the type of care to be provided. In general, an assessment of need should take into account not only the current and prospective health state of the individual, but also the ability of the patient to benefit from the possible procedures that might be offered. Doctors have a particular obligation to assess and weigh the needs of individual patients; doctors, managers and society, through some appropriate process, have a particular obligation to assess and weigh the needs of groups of patients and potential patients (12).

\section{GUIDELINES FOR DECISIONS AT THE AGGREGATE LEVEL}

5. Both justice and efficiency require universal access by the eligible population to basic health-care assessment in order that health-care needs may be evaluated (13).

6. Justice, if it is to include a component of concern to meet people's health-care needs, requires universal and equal access by the eligible population to basic health care.

7. The definition of the 'eligible population' should be determined through an appropriate process in accord with the goals of justice. The definition of 'basic services' and of the needs to be met by services defined as 'basic' should be determined through an appropriate process in accord with the goals of justice and efficiency (14).

8. The market should not be the primary determinant of access and priorities. Sometimes relatively unfettered market transactions can deliver costeffective health-care products and services but, even when market processes deliver efficiency, they often do so at the cost of justice. Moreover, efficiency as well as justice may be impaired by market transactions, since unfettered market transactions may paradoxically impair efficiency by promoting noneffective, but superficially attractive, health-care options.

\section{GUIDELINES FOR DECISIONS AT THE INTERMEDIATE LEVEL}

9. Resource allocation to decision-making units (such as hospitals or clinics) should be consistent with the guidelines for decisions at the aggregate level.

10. Resource allocation within such units ought to be consistent with the guidelines for decisions at the individual level. For example groups of patients with similar needs ought to receive similar treatment, while hospital departments serving similar needs ought to receive similar funding.

11. Doctors within such units whose resources are affected by decisions at a higher level ought to be consulted during the decision-making process.

\section{GUIDELINES FOR DECISIONS AT THE INDIVIDUAL LEVEL (CLINICAL PRACTICE)}

12. Patients who are alike in relevant respects ought to be treated in like fashion, ie, distribution of health care ought not to depend on morally irrelevant criteria such as race, religion, or gender.

13. Ability to pay, including insurance status, is an unjust basis for discrimination in access to basic medical care.

14. Whenever a decision to treat one patient is likely to deny treatment to another with a similar need, the consideration of the opportunity-cost of that decision is an appropriate ethical concern (15).

15. Merit is not a legitimate criterion for the allocation 
of health-care resources $(16,17)$.

16. Health-care professionals at the clinical level ought not to make any treatment decision that undermines legitimate attempts at a higher level to establish just and efficient allocation of resources (18).

17. As with all research that produces results that have bearing on clinical decisions, efficacy, effectiveness and cost-effectiveness studies ought always to be interpreted critically. Particular attention should be given to the value judgements used, explicitly or implicitly, in outcome and quality elements of the studies, to the scope of outcomes and costs considered, to the sensitivity of the results to changes in key assumptions, and to their relevance to the purpose in mind. Such critical awareness is not particular to costeffectiveness studies. It is no less important in assessing all research results that bear on clinical and practice-related decisions.

\section{Notes to Part IV}

(1) It is in the area of social policy concerning allocations of scarce health resources that international comparisons may be most instructive. Both the US and many European systems now inappropriately disguise the problem of scarcity and, in effect, purchase social stability at the price of justice. This occurs in the US by rendering invisible those who do not qualify for thirdpariy health coverage and who thus may be deprived of basic care; by denying the need for systematic trade-offs and tragic choices by focusing public attention on only one issue or one case at a time; and by avoiding sustained, systematic discussion of the 'ripple effects' of the allocation decision made in response to that one issue or case, for example, the denial of basic health care to others because the White House intervened to secure a liver transplant for a child out of the fixed resources of the Medicaid budget. To a large extent these issues are disguised because the US lacks any true system of providing health care equivalent to that in European nations. (Indeed, if the US adopted a system, these decisions could no longer be disguised, and it may be the reluctance to take any responsibility for such decisions that makes US politicians favour continuation of the present non-system.)

By contrast, European countries have systems, and explicit decisions are made by physicians and by administrators within these systems to allocate funds for some sorts of care and not for others. The failure of honesty arises when the reasons for such allocation decisions are not made explicit in public, so that individual patients cannot know when treatment is withheld because of lack of benefit and when for primarily economic reasons. This is exacerbated when doctors and administrators in different regions make quite different decisions in similar cases, so that which patients get care may depend as much on geography as on diagnosis. (Howard Brody)

(2) Scarcities troubling the health services in (peaceful) western societies are, morally speaking, significantly different from scarcities of medical resources on the battlefield, in circumstances of national disasters, and under conditions of extreme poverty. The guidelines we have proposed will have consequences for the three latter kinds of scarcity as well. But even under 'normal'

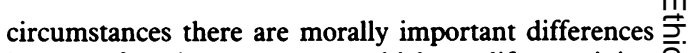

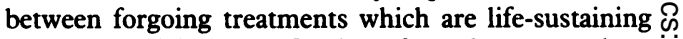
and those which are not. In view of our chosen mandate: to develop 'guidelines for decisions to forgo life- $\stackrel{\overrightarrow{7}}{+}$ prolonging medical treatment' (my emphasis), I do not feel that our guidelines in Part IV are sufficiently specific to $\frac{\bar{\sigma}}{\sigma}$ state, as we indicated in note 12, Part $I$, we would do, $\overline{\bar{c}}$ 'precisely what should be considered when drawing such $\overrightarrow{\mathbb{D}}$ lines'. (K E Tranoy)

(3) There are at least two radically different uses of the term \& health-care need. The standard sense of need is that one $\vec{O}$ needs $\mathrm{X}$ if one is in some way harmed in the absence of ? $X$. Similarly one has a health-care need for $X$ if without $\overrightarrow{\vec{\omega}}$ $\mathrm{X}$ one's health is harmed. Thus if one is dying from lung cancer, one needs a cure for the cancer.

On the other hand a contemporary account of need by $\overparen{\Phi}$ some health economists is that need means 'capacity to $\vec{\infty}$ benefit'. Thus if there is no effective treatment for a $i s$ particular cancer, according to this definition one cannot be said to 'need' any of the existing ineffectual treatments.

The distinction necessary here, whatever definition of $\vec{\sigma}$ need is used, is between having health-care needs and 음 having meetable health-care needs. From the $\vec{c}$ perspective of providers of health care there are two $\odot$ different sorts of decisions to be made: 1) who has the $\frac{\mathbb{D}}{\mathbb{D}}$ greatest health-care needs? and 2) how should the $\frac{\mathbb{D}}{3}$ available health-care interventions be distributed to $\bar{\partial}$ meet these needs?

(4) My own view, still under development, is that negd $\vec{\theta}$ creates a presumptive claim in justice even if the need cannot be met: and the greater the need(s) the greater the presumptive claim. On this view someone with a great unmeetable need (say for a cure for lung cancer) and a smaller but meetable need (say for relief of pain or $\bar{\partial}$ breathlessness) has a greater presumptive claim in justice to have his meetable need met than a person who has the $\frac{\circ}{D}$ same degree of meetable need (say pain or dyspnoea) but $\varrho$ who does not have the additional unmeetable need (say $\overrightarrow{\overrightarrow{0}}$ for a cure for lung cancer). This view fully accepts the 3 claim that one cannot need existing treatments that do $\supsetneq$ not meet one's needs. Indeed, it is surely an analytic 0 truth that one can only need that which would meet one's need. But the fact of having a need, even if it is for $\bar{D}$ something that is unavailable - ie the fact of having an unmeetable need - creates a prima facie claim in justice to 3 . have one's co-existing meetable needs met. This view is consistent with widespread medical and other $\frac{\mathrm{O}}{3}$ humanitarian tendencies to favour the sick over the less sick, for care and attention. But it is not consistent with 음 any medical tendency to give inappropriate but available $D$ treatments, ie treatments that will not produce net medical benefit, as a substitute for unavailable, perhaps $\overline{\mathrm{N}}$ non-existent, appropriate treatment(s). (Raanan ${ }^{\top}$ Gillon)

(5) The property principle recognises two types of resources used to purchase medical care: those which arise from $\omega$ publicly collected revenues (taxes) and those directly or indirectly generated from individuals (through fees ore insurance premiums). Each society must decide which $\frac{}{\Phi}$ approach to the payment for, and thus provision of, $\stackrel{\text { ? }}{+}$ medical care is appropriate. For those societies in which $\square$ public funds predominate, based on some socially $\bar{O}$ defined 'need' criteria, expenditure priorities should be set by cost-effectiveness analysis, with appropriate $\frac{\rho}{\mathbb{D}}$ provisos regarding the measurement of both cost and 2 effectiveness. The relevant decision-rule should be:ס 
offer all care for which the marginal social beneficial effects exceed the marginal social costs or rank the options by net social benefit. For those societies in which individual choice (of insurance plan, group of doctors, or hospitals) predominates, individual cost-utility analysis is the relevant decision-making framework. Doctors (or ethics groups) should advise patients (or their advocates) as to the potential effects (both cost and outcome) of each alternative, but decision-making and responsibility remain with each individual patient. (Merton D Finkler)

(6) Although both medical care and health care in the widest sense of the terms are perceived to make significant contributions to the quality of people's lives, not all medical care contributes to better health. Moreover, the contribution to better health and longer life made by improvements in lifestyle, housing, and public and personal hygiene may be greater than that of health care - especially in conditions of poverty.

(7) Some delegates felt that any ethically responsible allocation of scarce resources should consider this concept of demerit. Others felt strongly that to do so would re-introduce a most reactionary element into medical practice. See guideline 15 and note (16) below.

(8) Scarcity, by definition, requires choice. Any choice in the context of scarcity requires forgoing alternative choices. The opportunity-cost of any treatment, procedure or allocation is whatever could have been selected with the forgone choice. Societies may deny that they make such choices or disguise the ones they make, but they do so at the price of honesty, justice, and efficiency.

(9) I abstain from endorsing this section. The issues do not presently lend themselves to guidelines, resulting in simplifications with which I do not agree. For example, guideline 13 appears to require a practising doctor to accept an unlimited number of patients without regard to ability to pay. Guideline 15 appears to prohibit offering discretionary services, such as plastic surgery or psychotherapy for minor problems to patients who are able to pay if the same service is not offered to everyone. (Norman Fost)

(10) Doctors, patients, law-makers and the general public all ought to know the nature of the principles being applied, both for allocation decisions and for treatment decisions under conditions of scarcity. Several myths need to be dispelled, including the notion that it is a patient's right to receive every treatment available that may be of some potential benefit and the notion that a health-care system should be obliged to provide any such service. Programmes of public education are needed to alert the public to the facts about scarcity, the ethical difficulties involved in rationing health care, and the limitations on a doctor's clinical freedom that make it impossible to provide every available treatment. The recent successful co-operation between community health organisations and public television in several states in the US is exemplary of efficient and responsible education of this kind.

(11) The difference between efficacy and effectiveness relates to the circumstances in which clinical trials are conducted. Efficacy studies are generally performed at large teaching hospitals, research groups, or other 'centres of excellence'. They are designed to determine whether or not a particular procedure will achieve its intended biological work, ie whether or not it will work at all. Effectiveness studies, by contrast, attempt to assess the impact under normal operating conditions of adopting the procedure in question. Cost-effectiveness does not imply merely minimal cost; true costeffectiveness ought to take account of the full range of opportunity-costs and of all relevant outcomes, whether good or bad.

(12) It is difficult for doctors to weigh at the clinical level the claims of patients for whom they have no direct responsibility. Such judgements ought to be made explicitly at higher levels of decision-making authority, ie, at the aggregate or intermediate levels, with appropriate participation of the doctors affected by such decisions. Our distinction between levels of decisionmaking is not intended to imply that it is always possible to distinguish clearly between 'medical' and 'social' decisions. Decisions at the clinical level must often, and quite properly, take account of the individual personal, family, and social circumstances of a patient, for example in deciding when to discharge the patient from the hospital. The higher-level decisions must also, and quite properly, consider the medical benefits that may follow or that may be denied by clinical decisions at the patient level, operating within the resource constraints that are being determined at that higher level.

(13) If the accepted definition of justice includes a component of concern for the health-care needs of all, justice requires universal access to initial diagnostic services in order that need may be assessed; subsequent access and utlisation might depend on need and could, therefore, be selective. Efficiency, if by efficiency is meant the use of available resources so as to maximise the health of the eligible population, requires universal access to basic-needs assessment as well as subsequent selective use of programmes of care.

(14) Who is to be eligible ought to be governed by consideration of justice. Efficiency in the sense of community-health maximisation out of available resources suggests that the eligible population ought to be defined broadly rather than narrowly. What basic services can be afforded will depend, in part, on the opportunity-cost of devoting more resources to health care rather than to other sources of human welfare or flourishing. Such preferences are contingent both on their historical context and on the cultural values of societies, which in some jurisdictions may, of course, be quite heterogeneous. Cf The President's commission for the study of ethical problems in medicine and biomedical and behavioral research and Securing access to health care: a report on the ethical differences in the quality of health services. Washington DC: 1983; supt of docs; USGPO; vol 1: 18-46.

Some delegates wanted to attempt to describe standards for a 'decent basic minimum' for health care that all societies trying to be just and efficient should provide to all citizens. The analogy appealed to was the similar standards that most societies set for food and shelter. This effort, however, seemed fraught with difficulty. It seems to be impossible to define such standards in a way that is appropriate to all societies, regardless of culture and wealth. In other fields, such as housing, it may be possible to define an agreed minimum of 'shelter from the environment'. In medical care, however, such a threshold is difficult to establish in financial terms but might be based, perhaps, on degrees of effectiveness or by distinguishing between effective medicine and the adjunct services provided, such as the hotel services of hospitals. For example, justice in the consumption of 
relatively ineffective care and in the hotel dimensions of a hospital's services may be deemed to be of no greater distributive concern than the consumption of the hotel services of hotels, and of much less concern than the consumption of needed medical care. Some delegates also argued for a concept of acceptable maxima rather than minima.

(15) Doctors, particularly when pressing for more or better resources for the care of the specific patients for whom they are responsible, ought also to bear in mind that similar claims from other doctors on behalf of other patients may be in conflict with their claims. Resolution of any such conflicts, when known, should be accomplished in an open process consistent with the goals of justice and efficiency.

(16) Delegates were unamimous on this point. The main reasons for holding this view are 1) the impossibility of identifying any pathological state which did not have the behaviour of the person in question as a partial determinant; 2) the awareness that socialisation processes in different parts of society (and, of course, across societies) may substantially reduce the autonomy of individuals and, hence, the appropriateness of classifying behaviours as 'meritorious'. Some delegates, however, expressed the position that it is not necessarily inconsistent with justice or efficiency for those who have knowingly contributed to their own ill-health to be called on to pay more for their care than might otherwise be required by the particular health-care system. The majority of delegates opposed even this form of 'demerit' based allocation suggesting that: a) there are better ways of requiring those in question to contribute to the cost of their care (for example, by taxation of high-risk ? behaviour) and b) that setting the payment for high-risk behaviour at the point of health care undermines the value of community solidarity on which many healthcare delivery-systems are based.

(17) Merit, or social worth, in disaster conditions and in the military, in terms of benefiting many people is a valid moral consideration. In civil medicine, merit should not routinely enter into the decision-making process in triage. This, however, is not because there is a social $\overrightarrow{0}$ consensus that this would always be unethical. Rather it is because in practice there is no agreement on the $\vec{\omega}$ relative weighting to be assigned to different kinds of social contributions. Hence, in exceptional well-defined situations, merit may bear weight and ought to be considered. (Avraham Steinberg)

(18) Legitimate decisions from a higher level must presumably be 'imposed' on doctors at the clinical level with some measure of legal force. It remains the duty of the doctor to offer everything of benefit to his patient $\vec{\sigma}$ unless it is proscribed by society. When it is proscribed 음 by society, the decision should not be disguised as a medical decision. To do so would be deception both on $\vec{\sim}$ the part of society and the doctor. Patients ought to be aware that decisions are politico-economic when they are, so that they may protect themselves appropriately in the political arena. (Frederick R Abrams) 